\title{
Neuroimaging provides insights into cognitive dysfunction in MS
}

Gradual loss of cognitive function is common in patients with multiple sclerosis (MS), but traditional MRI measures of disease progression yield limited information about cognitive impairment. Now, two new studies suggest that subclinical disease activity, and changes in thalamic structure and function are good predictors of cognitive performance.

Menno Schoonheim and colleagues from the VU University Medical Center, Netherlands, focused their search for MRI correlates of cognitive function on the thalamus - a brain area best known for its importance for sensory processing.

"Atrophy of the thalamus is the MRI measurement that has been most strongly and robustly related to cognitive dysfunction in MS," Schoonheim explains. Besides structural measures, the investigators also looked into functional connectivity between the thalamus and cortical areas in a cohort of 157 patients with MS and 47 healthy controls.

The researchers discovered that thalamic volume was reduced and mean diffusivity was increased in all patients with MS - and particularly in those with severe cognitive impairment. Interestingly, functional connectivity between the thalamus and multiple cortical regions was found to be increased in the patients with severe cognitive impairment.

"We interpreted our findings as a maladaptive increase in functional connectivity," comments Schoonheim. "Thalamic volume was already reduced in cognitively preserved patients, but patients with severe cognitive impairment were the only group to show increased functional connectivity."

In another study, Alfredo Damasceno and co-workers from the University of Campinas, Brazil, evaluated the number of asymptomatic gadoliniumenhancing lesions-a measure of subclinical disease activity in MS-as potential predictor of cognitive dysfunction.

\section{These studies highlight the complexity that underlies cognitive deficits in MS 77}

"Gadolinium-enhancing lesions can be associated with increased burden of cortical and white matter lesions, which can also affect cognition, so we decided to investigate whether subclinical disease activity independently contributed to cognitive decline," says Damasceno.

The researchers scanned 42 patients with relapsing-remitting MS and 30 healthy controls with 3 T MRI. All participants also underwent cognitive testing.

$15 \%$ of the patients showed subclinical disease activity on MRI, and these patients had the worst cognitive performance. Cortical lesion burden and gadolinium-enhancing lesions were the main predictors of cognitive deficits.

These studies highlight the complexity that underlies cognitive deficits in MS. "Together, these findings corroborate the dire need for longitudinal cognitive studies using moreadvanced imaging measures," concludes Schoonheim.

\section{Hemi Malkki}

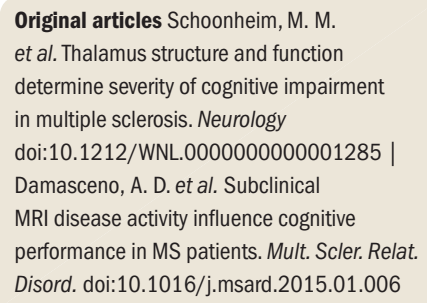

